\title{
A Simple Grid-Voltage-Sensorless Control Scheme for PFC Boost Converters
}

\author{
Cong-Long Nguyen*, Hong-Hee Lee ${ }^{\dagger}$, and Tae-Won Chun* \\ ${ }^{*}$ School of Electrical Engineering, University of Ulsan, Ulsan, Korea
}

\begin{abstract}
This paper introduces a simple grid-voltage-sensorless control scheme for single-phase power factor correction (PFC) boost converters. The grid voltage waveform is obtained based on the dc output voltage, the switching duty ratio, and a phase-lead compensator. In addition, the duty ratio feedback is utilized to obtain the unity input power factor and the zero harmonic current. The proposed control scheme is designed and mathematically analyzed based on a small-signal model of PFC boost converters. To verify the effectiveness of the proposed control scheme, several simulations and experiments are carried out in two applications: an industrial power system with a $60 \mathrm{~Hz}$ grid frequency and a commercial aircraft application with a $400 \mathrm{~Hz}$ grid frequency.
\end{abstract}

Key words: Power factor correction (PFC) techniques, PFC boost converters, Sensorless control, Small-signal model

\section{INTRODUCTION}

Currently, ac-to-dc conversion systems are widely used to supply power from the ac grid for various applications, such as battery chargers, electronic ballasts, household electric equipment, switch-mode power supplies (SMPS), uninterrupted power supplies (UPSs), and motor drives [1], [2]. However, conventional ac-to-dc converters based on diode or thyristor rectifiers draw pulsed currents from the utility grid. These pulsed currents cause severe problems such as voltage distortion, a poor input power factor, and electromagnetic interference due to harmonic components [3]. Therefore, several standards and regulations have been introduced to limit the current harmonics to within acceptable levels [4], [5].

The power factor correction (PFC) method for an ac-to-dc conversion system is an attractive solution that complies with various standards and regulations. There have been several studies published recently that focus on PFC converter topologies. For example, an integrated buck-flyback PFC converter was introduced in [6], the authors of [7] proposed a new bridgeless Cuk rectifier for PFC applications, and several configurations based on PFC boost converters were investigated in [8]. High power density, high efficiency, and low cost are the three main advantages that make PFC boost

Manuscript received Feb. 19, 2014; accepted May 28, 2014

Recommended for publication by Associate Editor Marian P. Kazmierkowski.

${ }^{\dagger}$ Corresponding Author: hhlee@mail.ulsan.ac.kr

Tel: +82-52-259-2187, Fax: +82-52-259-1686, University of Ulsan

* School of Electrical Engineering, University of Ulsan, Korea converters popular in commercial products [9]. Fig. 1 shows the most typical PFC boost converter and its control scheme to obtain a desired dc output voltage with a unity input power factor. Although the conventional control scheme is easy to implement in both digital and analog systems, it has several drawbacks, including phase leading in the grid current and zero-crossing distortion [10]-[13].

To enhance the performance of the conventional control scheme of PFC boost converters, some studies have introduced new control algorithms to achieve a low total harmonics distortion (THD) and a high input power factor (PF). In [10] and [11], a notch-filter was used to improve the control dynamic response and to reduce the grid current distortion during load variations. The authors of [12] applied a washout filter to stabilize the boost PFC converters by suppressing the period-doubling bifurcation at the line frequency. The authors of [13] inserted an adaptive first-order low-pass filter into the grid voltage measuring circuit to solve the zero-crossing distortion phenomena and the grid-current phase leading issue. In addition, feed-forward techniques were applied to the current control loop, including the duty-ratio feed-forward [14] and current feed-forward methods [15], which resulted in a unity input power factor and guaranteed a low harmonic content in the grid current.

Despite the improved control methods introduced in [10]-[15], these approaches still pose some disadvantages, such as large computation times, noise and error issues during the measurement of the control variables, and high costs due to the requirement of a large number of sensors (two voltage sensors 


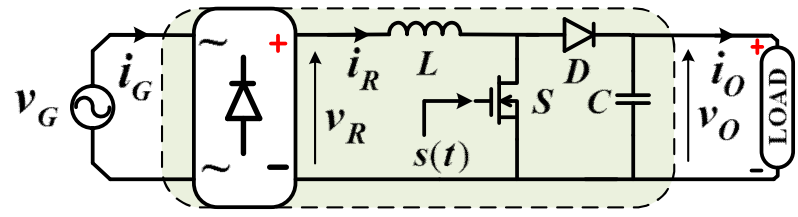

(a)

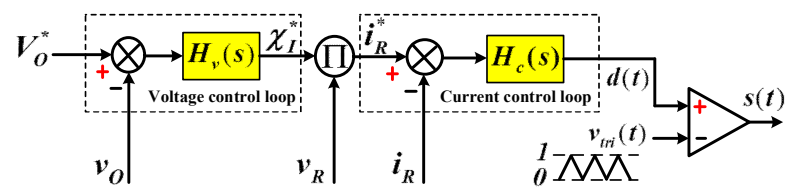

(b)

Fig. 1. (a) PFC boost converter configuration. (b) Conventional control scheme for the PFC boost converter.

and one current transducer). To overcome the noise and error issues and to reduce the control system cost, sensorless control techniques have been developed for PFC boost converters [16]-[22]. However, these sensorless control schemes require many added calculation steps to estimate or predict the desired control variables, and the control performance depends significantly on the accuracy of the converter parameters. For example, in [16], even though the proposed control scheme was made simple by having no sensors to detect the grid current or dc output voltage, its dynamic response was quite poor, and the control system did not consider the effects of converter parameter variations. In [17]-[20], both single-loop and multi-loop grid current senseless controls were introduced by considering the effects of the difference between the nominal and real circuit parameters. In addition, a nonlinear carrier control technique that allows the PFC boost converters to operation under continuous conduction mode without grid voltage sensing was developed in [21] and [22]. However, these senseless control schemes are not simple and they are not low cost because specialized devices such as the field-programmable gate array or external analog devices are required to implement the control algorithms.

In this paper, a simple control scheme for a PFC boost converter is developed without the grid voltage sensor. The grid voltage is detected indirectly from the dc output voltage, the switching duty ratio, and a phase-lead compensator. Simplicity and no serious influence of the converter parameters on the control performance are two merits of the proposed grid-voltage detecting method. Furthermore, with the duty-ratio feedback, the proposed control scheme is able to obtain a unity input power factor and inject zero harmonic current to the grid in both $60-\mathrm{Hz}$ industrial power systems and $400-\mathrm{Hz}$ commercial aircraft applications. The stability of the proposed control scheme is verified mathematically based on a small signal model of the converter.

This paper is organized as follows. In Section II, a grid-voltage model under the unity input power factor condition is constructed, which demonstrates that the

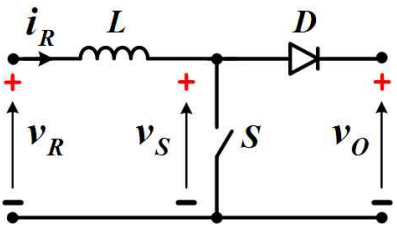

(a)

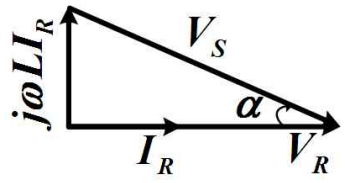

(b)
Fig. 2. (a) Major components and signals in PFC boost converter. (b) Phasor diagram of rectified grid voltage and switch voltage.

grid-voltage waveform can be detected without voltage sensor. Next, the proposed control scheme and its performance are theoretically analyzed in Section III. To verify the efficacies of the proposed control scheme, several simulations and experiments are carried out, and their results are presented in Sections IV and V, respectively. Finally, in Section VI, some conclusions are provided regarding the contributions of this paper.

\section{GRID VOLTAGE DETECTION}

As shown in Fig. 1(a), the PFC boost converter is composed of a full bridge diode rectifier, an active-passive switch pair, and energy storage elements including a capacitor and an inductor. Switch $S$ regulates the rectified grid current $i_{R}$ to be in phase with the rectified grid voltage $v_{R}$ so as to achieve a unity input power factor. To create the grid current reference, conventional control schemes usually need to detect the grid voltage waveform through a voltage sensing circuit. However, additional sensors complicate the system and increase the system cost. To resolve these problems, this paper proposes a control scheme without a grid voltage sensor by considering the relationship between the grid voltage $v_{G}$ and the switch voltage $v_{S}$, where $v_{S}$ is proportional to the switch duty ratio and the dc output voltage.

Assuming that the converter operates under the unity power factor condition with a pure sinusoidal grid voltage, the grid voltage and grid current can be expressed as:

$$
v_{G}(t)=\hat{V}_{G} \sin (\omega t), \quad i_{G}(t)=\hat{I}_{G} \sin (\omega t),
$$

where $\omega$ is the grid angular frequency, and $\hat{V}_{G}$ and $\hat{I}_{G}$ are the magnitudes of the grid voltage and grid current, respectively. Fig. 2(a) shows the key components of the PFC boost converter, where the grid voltage and grid current after rectification are $v_{R}$ and $i_{R}$, respectively. By using the phasor diagram in Fig. 2(b), the switch voltage can be derived as follows:

$$
V_{S}=V_{R}-j \omega L I_{R} .
$$

During the positive haft period of the grid voltage, the rectified grid voltage and current are identical to the grid voltage and current, respectively (i.e., $v_{R}=v_{G}$ and $i_{R}=i_{G}$ ). Thus, the switch voltage can be obtained from (1) and (2): 


$$
v_{S}(t)=\hat{V}_{S} \sin (\omega t-\alpha)
$$

where:

$$
\begin{gathered}
\hat{V}_{S}=\hat{V}_{G} \sqrt{1+L^{2} \omega^{2} \chi_{\mathrm{I}}^{2}}, \\
\alpha=\arctan \left(L \omega \chi_{\mathrm{I}}\right), \\
\chi_{\mathrm{I}}=\frac{i_{G}}{v_{G}}=\frac{\hat{I}_{G}}{\hat{V}_{G}} .
\end{gathered}
$$

When compared with the rectified grid voltage, the switch voltage has a phase lag but a greater magnitude, as illustrated in Fig. 2(b). From (4) and (5), both the phase lag $\alpha$ and the magnitude $V_{S}$ depend on the inductor, the grid frequency, and the input conductance $\chi_{I}$. Fig. 3 shows the phase lags caused by the inductor for two $60 \mathrm{~Hz}$ and $400 \mathrm{~Hz}$ grid systems. These are typical frequencies for industrial power systems and for commercial aircraft applications, respectively, when the input conductance is fixed at $0.3 \widetilde{\sigma}$. It can be seen that the phase lag becomes more significant when the system operates at a higher grid frequency.

From Fig. 2(a), the instantaneous voltage across the inductor can be given as:

$$
v_{L}(t)=\left\{\begin{array}{lc}
v_{R}(t), & t_{0} \leq t \leq t_{0}+d T_{S} \\
v_{R}(t)-v_{O}(t), & t_{0}+d T_{S} \leq t \leq t_{0}+T_{S}
\end{array}\right.
$$

where $t_{0}$ represents the time when the switch starts to turn on, and $d$ denotes the switching duty ratio. The average inductor voltage $\bar{v}_{L}$ during the switching period can be derived by:

$$
\begin{aligned}
\bar{v}_{L}\left(t_{0}\right) & =\frac{1}{T_{S}} \int_{t_{0}}^{t_{0}+T_{S}} v_{L}(\tau) d \tau \\
= & \frac{1}{T_{S}}\left[\int_{t_{0}}^{t_{0}+d T_{S}} v_{R}(\tau) d \tau+\int_{t_{0}+d T_{S}}^{t_{0}+T_{S}}\left\{v_{R}(\tau)-v_{O}(\tau)\right\} d \tau\right] .
\end{aligned}
$$

Because the switching period $T_{S}$ is very small, the grid and output voltages are assumed to be constant during $T_{S}$. Then, (8) can be simplified as follows:

$$
\bar{v}_{L}\left(t_{0}\right)=v_{R}\left(t_{0}\right)-(1-d) v_{O}\left(t_{0}\right) .
$$

In addition, using the Kirchhoff voltage law, the switch voltage can be determined simply by:

$$
v_{S}\left(t_{0}\right)=v_{R}\left(t_{0}\right)-\bar{v}_{L}\left(t_{0}\right) .
$$

From (9) and (10), the relationship between the output voltage and the switch voltage can be expressed as:

$$
v_{S}=(1-d) v_{O} \text {. }
$$

By using (11), the switch voltage $v_{S}$ can be determined from the output voltage $v_{O}$, and then the grid voltage can be determined from the switch voltage based on (4) and (5). Although the grid voltage can be calculated mathematically from (4), (5) and (11), this takes a long time due to the

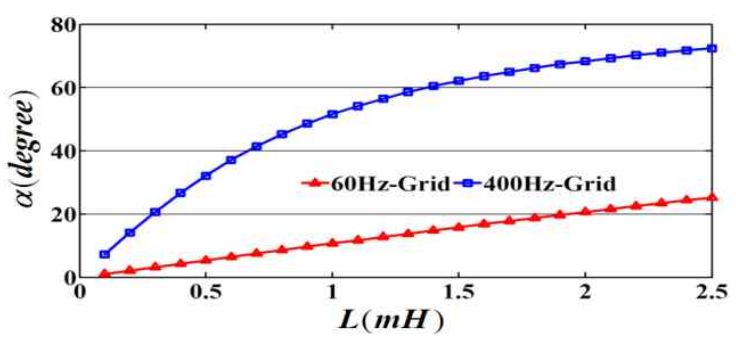

Fig. 3. Phase lag of the switch voltage compared with the grid voltage.

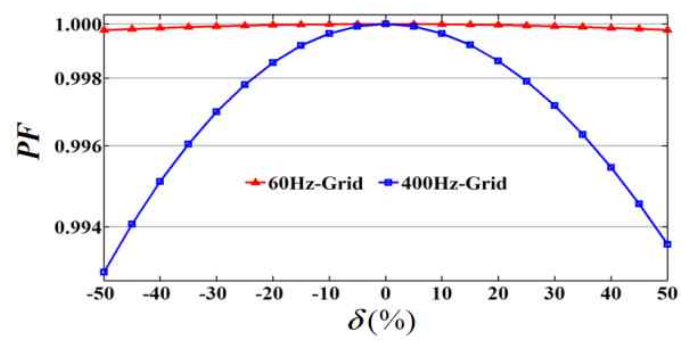

Fig. 4. The inductance variation effect.

complexity of the process. An alternative way that facilitates the grid voltage detection is to use a leading phase compensator, which compensates for the differences in the phase lag and the magnitudes between the switch voltage and the grid voltage. By utilizing a leading-phase compensator, the grid voltage waveform can be identified from the switch voltage. In this paper, a first-order leading-phase compensator applied for a simple design is:

$$
H_{\mathrm{Com}}(s)=\frac{1+L \chi_{I} s}{1+L^{2} \omega^{2} \chi_{I}^{2}} .
$$

To investigate the effects of inductor parameter variations in (12), the deviation between the nominal inductance and its real value is investigated. From (5) and (12) and by using basic trigonometric tangent functions, the phase error between the estimated grid voltage and its real value can be expressed by:

$$
\Delta \alpha=\arctan \frac{\delta L_{N} \omega \chi_{I}}{1+(1+\delta) L_{N}^{2} \omega^{2} \chi_{I}^{2}},
$$

where $\delta$ denotes the deviation ratio between the nominal value $L_{N}$ and the real value $L_{R}$ of the inductor:

$$
\delta=\frac{L_{R}-L_{N}}{L_{N}} 100 \% .
$$

Under the assumption that the system controller tracks its reference successfully, the input power factor can be expressed as a cosine function of the error phase $\Delta \alpha$ or $P F=\cos (\Delta \alpha)$. To evaluate the effects of inductance variations on the converter performance, Fig. 4 shows the input power factor in accordance with inductance variations with a nominal value $L_{N}=0.5 \mathrm{mH}$ at $\chi_{I}=0.3 \%$. The PF decreases when the inductance variation is large, and this effect is obvious at high 


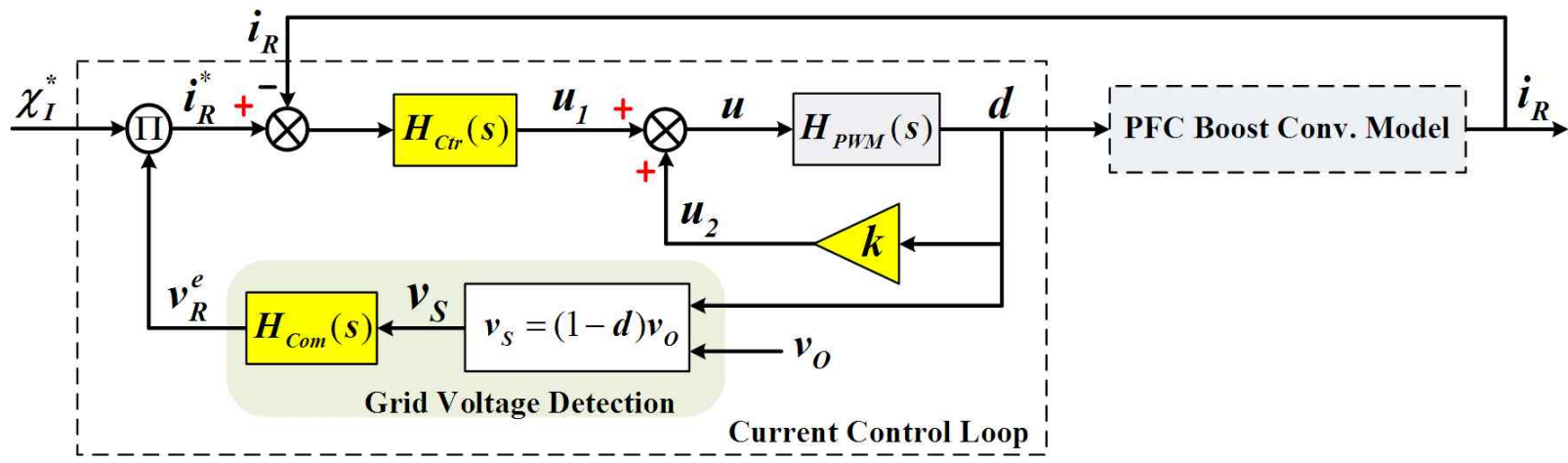

Fig. 5. Grid-voltage sensorless current control loop with duty ratio feedback.

grid frequencies. However, the PF is always higher than 0.99, although the inductance variation reaches $50 \%$, which verifies that the detecting grid voltage method is not seriously affected by the converter parameter variations.

\section{GRID-VOLTAGE-SENSORLESS POWER FACTOR CONTROL WITH DUTY-RATIO FEEDBACK}

In Fig. 1, the objective of the PFC boost converter is to regulate the output voltage at a desired dc value and to achieve the unity input power factor and low harmonic content of the grid current. In Fig. 1(b), a voltage control loop creates the desired input conductance $\chi_{I}^{*}$ to obtain the current reference. Because the reference of the voltage control loop is a dc signal, its performance does not severely depend on the controller bandwidth or the sampling frequency. Therefore, a simple controller, such as a proportional-integral (PI) compensator, is usually adopted [14]. Meanwhile, the current reference is a sinusoidal command. Thus, a PI controller alone cannot guarantee that the grid current will track well to its reference [15]. In this paper, a current controller suitable for the grid voltage estimation is developed by inserting a duty ratio feedback signal.

Fig. 5 shows a block diagram of the proposed grid-voltagesensorless current controller. To implement the PFC control system without a grid voltage sensor, the rectified grid voltage is estimated from the output voltage and the phase compensator. As shown in Fig. 5, the feedback signal from the output of the PWM modulator is applied with a gain $k$ to effectively obtain a unity input power factor.

To design an effective current controller, the converter is analyzed by using the circuit averaging method so that the converter is represented by a linear circuit model [23]. In the averaging method, the state variables are divided into two components: a small-signal value denoted by a lowercase letter with a tilde and a steady-state dc value expressed by an uppercase letter:

$$
d=D+\tilde{d}
$$

$$
\begin{aligned}
& i_{R}=I_{R}+\tilde{i}_{R}, \\
& v_{R}=V_{R}+\tilde{v}_{R}, \\
& v_{O}=V_{O}+\tilde{v}_{O} .
\end{aligned}
$$

The small-signal model is dependent on the system operating point which is regarded as the dc component of each variable: $V_{O}$ is the dc component of the output voltage, $V_{R}$ and $I_{R}$ are the root-mean squares (RMS) of the steady state component of the grid voltage and grid current, respectively, and $D$ is defined as $D=1-V_{R} / V_{O}$. By using the state variables defined in (15)-(18), the rectified grid current obtained from (9)-(11) is written as:

$$
i_{R}=\frac{1}{s L} V_{R}-\frac{(1-D)}{s L} V_{O}+\frac{1}{s L} \tilde{v}_{R}+\frac{V_{O}}{s L} \tilde{d}-\frac{1-D}{s L} \tilde{v}_{O} .
$$

As shown in (19), $i_{R}$ is composed of two components, the steady-state dc value and the small-signal component. The small-signal component is defined as:

$$
\tilde{i}_{R}=\frac{1}{s L} \tilde{v}_{R}+\frac{V_{O}}{s L} \tilde{d}-\frac{1-D}{s L} \tilde{v}_{O} .
$$

From Fig. 5, the small-signal component of the control term $u_{1}$ can be derived as follows:

$$
\begin{aligned}
\tilde{u}_{1}= & \chi_{I}^{*}(1-D) H_{C o m}(s) H_{C t r}(s) \tilde{v}_{O} \\
& -\chi_{I}^{*} V_{O} H_{C o m}(s) H_{C t r}(s) \tilde{d}-H_{C t r}(s) \tilde{i}_{R} .
\end{aligned}
$$

In addition, the total control term $\tilde{u}$ becomes:

$$
\tilde{u}=\left(k \tilde{d}+\tilde{u}_{1}\right) H_{P W M}(s) .
$$

By substituting (21) into (22), the duty ratio is defined as:

$$
\begin{aligned}
\tilde{d}= & \frac{\chi_{I}^{*}(1-D) H_{C o m}(s) H_{C t r}(s) H_{P W M}(s)}{1-k H_{P W M}(s)+\chi_{I}^{*} V_{O} H_{C o m}(s) H_{C t r}(s) H_{P W M}(s)} \tilde{v}_{O} \\
& -\frac{H_{C t r}(s) H_{P W M}(s)}{1-k H_{P W M}(s)+\chi_{I}^{*} V_{O} H_{C o m}(s) H_{C t r}(s) H_{P W M}(s)} \tilde{i}_{R} .
\end{aligned}
$$


From (20) and (23), the following equation can be obtained:

$$
\begin{aligned}
& \left(s L+\frac{V_{O} H_{C t r}(s) H_{P W M}(s)}{1-k H_{P W M}(s)+\chi_{I}^{*} V_{O} H_{C o m}(s) H_{C t r}(s) H_{P W M}(s)}\right) \tilde{i}_{R} \\
& =\tilde{v}_{R}+\left[\frac{\chi_{I}^{*}(1-D) V_{O} H_{C o m}(s) H_{C t r}(s) H_{P W M}(s)}{1-k H_{P W M}(s)+\chi_{I}^{*} V_{O} H_{C o m}(s) H_{C t r}(s) H_{P W M}(s)}\right. \\
& -(1-D)] \tilde{v}_{O} .
\end{aligned}
$$

According to (24), the grid current $\tilde{i}_{R}$ depends on two input signals: the grid voltage $\tilde{v}_{R}$ and the output voltage $\tilde{v}_{O}$. Therefore, the proposed current control scheme can be evaluated by taking into account the relationship between the grid current and the grid voltage in (25), and the relationship between the grid current and the output voltage in (26). The relationship in (25) shows the input conductance response of the PFC control system, while (26) indicates the grid current disturbance caused by output voltage variations. In order to satisfy the control criteria, which requires a constant conductance $\chi_{I}^{*}$ and a pure sinusoidal grid current, (25) should be constant and (26) should be zero.

To investigate the control performance, the transfer functions shown in (25) and (26), such as $H_{C o m}(s), H_{C t r}(s)$, and $H_{P W M}(s)$, must be defined. The phase compensator $H_{C o m}(s)$ is defined in (12), and the controller $H_{C t r}(s)$ is:

$$
H_{C t r}(s)=K_{P}+\frac{K_{I}}{s},
$$

where $K_{P}$ and $K_{I}$ are the controller gains. The modulator block, denoted by $H_{P W M}(s)$, is a uniformly sampled symmetric-ontime modulator [24]. Its model in the frequency domain, taking into account one switching cycle delay, can be expressed as:

$$
H_{P W M}(s)=\frac{1}{2}\left(e^{-s \frac{1-D}{2} T_{s}}+e^{-s \frac{1+D}{2} T_{s}}\right) e^{-s T_{s}}
$$

Looking inside the disturbance caused by output voltage variation shown in (26), the numerator is approximately a negative constant if no duty ratio feedback is applied (i.e., $k=0$ ). In this case, the disturbance can be eliminated by choosing the highest possible gain of the controller $H_{C t r}(s)$. In the conventional control scheme, the disturbance can be reduced by using a large output capacitor to curtail output voltage variations. However, this makes the system dynamic response slow. When the duty ratio feedback is employed with a unity gain (i.e., $k=1$ ), the disturbance becomes almost zero. Therefore, the disturbance can be eliminated regardless of the controller property and the smoothing output capacitor. For more clearance, the system operation under the following condition is considered:

$$
\left\{0<\omega<\frac{K_{I}}{K_{P}}, T_{S} \ll \frac{K_{P}}{K_{I}} \text {, and } \frac{1}{L \chi_{I}^{*}} \gg \frac{K_{I}}{K_{P}}\right\},
$$

This leads to an approximation of the disturbance as follows:

$$
\frac{\tilde{i}_{R}}{\tilde{v}_{O}} \approx \frac{(1-D)(k-1)}{j \omega L(1-k)+\chi_{I}^{*} V_{O} K_{I}-j \omega V_{O} K_{P}} .
$$

Obviously, the disturbance can be canceled out if $k=1$.

The most important aspect to be taken into consideration is the input conductance expressed in (25). Under the circumstances defined in (29), the input conductance can be approximated by:

$$
\chi_{I}=\frac{\tilde{i}_{R}}{\tilde{v}_{R}} \approx \frac{j \omega(1-k)+\chi_{I}^{*} V_{O} K_{I}}{V_{O} K_{I}}=j \omega \zeta(1-k)+\chi_{I}^{*},
$$

where $\zeta=\left(V_{O} K_{I}\right)^{-1}$. It can be seen that when no duty ratio feedback path is applied, the input conductance has a positive real part and a positive imaginary part, indicating a resistive capacitive behavior. In other words, the grid current leads the grid voltage, resulting in a non-unity input power factor. On the other hand, with the duty ratio feedback, the input conductance acts as a pure resistive load and perfectly follows its reference $\chi_{I}^{*}$. Thus, a PFC boost converter using the proposed control scheme results in an input unity power factor and behaves as a resistive load over a wide frequency range. This ensures superior behavior from the PFC system.

Fig. 6 shows a Bode diagram of the input conductance of the PFC boost converter both with and without duty ratio feedback

$$
\begin{gathered}
\chi_{I}=\frac{\tilde{i}_{R}}{\tilde{v}_{R}}=\frac{1+\left(\chi_{I}^{*} V_{O} H_{C o m}(s) H_{C t r}(s)-k\right) H_{P W M}(s)}{\left[1-\left(k-\chi_{I}^{*} V_{O} H_{C o m}(s) H_{C t r}(s)\right) H_{P W M}(s)\right] s L+V_{O} H_{C t r}(s) H_{P W M}(s)} \\
\frac{\tilde{i}_{R}}{\tilde{v}_{O}}=\frac{(1-D)\left(k H_{P W M}(s)-1\right)}{\left[1-\left(k-\chi_{I}^{*} V_{O} H_{C o m}(s) H_{C t r}(s)\right) H_{P W M}(s)\right] s L+V_{O} H_{C t r}(s) H_{P W M}(s)}
\end{gathered}
$$




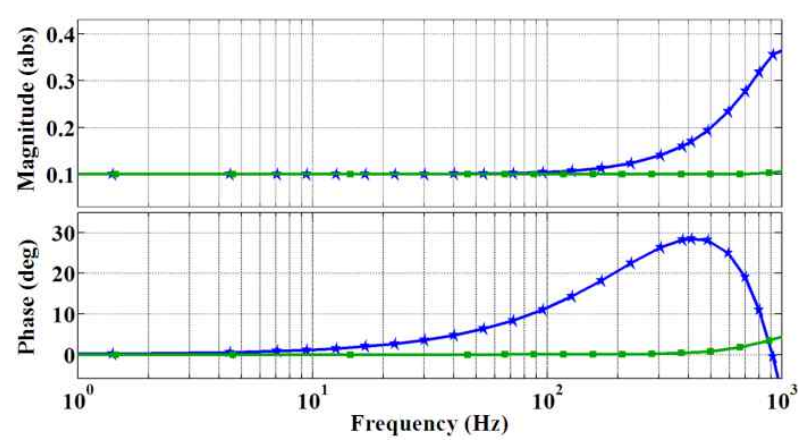

Fig. 6. Input conductance of the PFC boost converter: with the duty ratio feedback (the line with rectangle signs) and without the duty ratio feedback (the line with stars).

in the control scheme. To calculate the input conductance in (25), many parameters are required. They are chosen in a similar way to those of the simulation and experimental setup:

$$
\left\{\begin{array}{l}
\hat{V}_{G}=155 \mathrm{~V}, V_{O}=300 \mathrm{~V}, L=0.8 \mathrm{mH}, \chi_{I}^{*}=0.1 \mathrm{~J} \\
T_{s}=20 \mu \mathrm{s}, K_{P}=0.015, \text { and } K_{I}=50.0
\end{array}\right\}
$$

For the control scheme without duty ratio feedback, the magnitude of the input conductance only satisfies its reference in the low frequency range. However, at high frequencies, the magnitude responses are greater than the desired value. Furthermore, the phase performance of the input conductance cannot be kept at zero, causing a non-resistive input conductance in the PFC system. However, when duty ratio feedback is applied, the magnitude tracks its reference successfully, and the phase is always managed at zero in all frequency ranges. This guarantees that a pure resistive load is connected to the grid.

\section{Simulation RESUlts}

The proposed grid-voltage-sensorless control scheme is simulated using PSIM to verify its effectiveness. The system performance is evaluated at both a $60-\mathrm{Hz}$ industrial grid frequency and at a $400-\mathrm{Hz}$ commercial aircraft utility frequency. Table I shows the ideal and practical system parameters used in the simulation. The simple PI controller together with a notch-filter is used as the outer voltage controller to create the input conductance command.

For the $60-\mathrm{Hz}$ grid power source, Fig. 7 shows the simulation results when the ideal system parameters are applied to obtain the output voltage $V_{O}^{*}=300 \mathrm{~V}$ with $\chi_{I}^{*}=0.093 \approx$. The estimated and real rectified grid voltages together with the estimation error are plotted in Fig. 7(a). It can be seen that the rectified grid voltage is detected perfectly without the sensing grid voltage. In Fig. 7(b), the grid voltage, the grid current, and the output voltage are illustrated. Referring to Fig. 7(b), the grid current satisfies the command
TABLE I

SYSTEM PARAMETERS

\begin{tabular}{|c|c|c|}
\cline { 2 - 3 } \multicolumn{1}{c|}{} & Ideal & Practical \\
\hline Grid voltage (peak) & $\hat{V}_{G}=155 \mathrm{~V}$ & $\hat{V}_{G}=155 \mathrm{~V}$ \\
\hline \multirow{2}{*}{ Smoothing inductor } & $L=0.8 \mathrm{mH}$ & $L=0.8 \mathrm{mH}$ \\
& $R_{L}=0 \Omega$ & $R_{L}=0.18 \Omega$ \\
\hline \multirow{2}{*}{ Smoothing capacitor } & $C=2200 \mu \mathrm{F}$ & $C=2200 \mu \mathrm{F}$ \\
& $R_{C}=0 \Omega$ & $R_{C}=48 \mathrm{~m} \Omega$ \\
\hline \multirow{3}{*}{ Switch (MOSFET) } & $R_{D S}=0 \Omega$ & $R_{D S}=0.22 \Omega$ \\
& $V_{S D}=0 \mathrm{~V}$ & $V_{S D}=0.7 \mathrm{~V}$ \\
& $V_{F}=0 \mathrm{~V}$ & $V_{F}=1.5 \mathrm{~V}$ \\
\hline \multirow{2}{*}{ Diode } & $R_{D}=0 \Omega$ & $R_{D}=12 \mathrm{~m} \Omega$ \\
& $V_{F}=0 \mathrm{~V}$ & $V_{F}=1.6 \mathrm{~V}$ \\
\hline Load & $80 \Omega$ & $80 \Omega$ \\
\hline
\end{tabular}

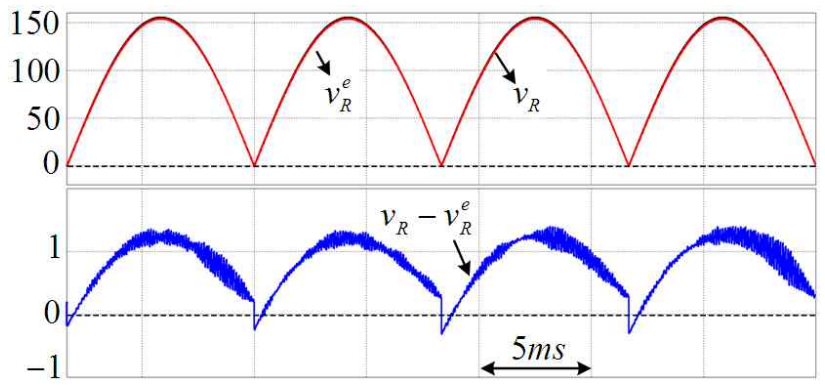

(a)

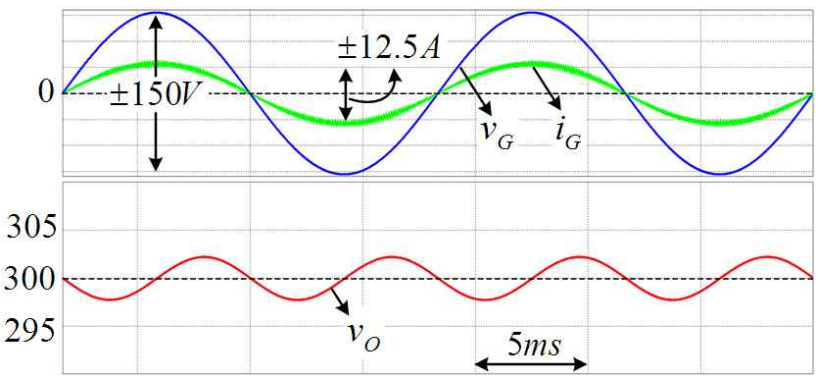

(b)

Fig. 7. Simulated waveforms for ideal circuit elements with a 60-Hz-grid power supply. (a) Estimated rectified grid voltage and estimation error. (b) Grid voltage, grid current, and output voltage.

and produces a sinusoidal waveform in phase with the grid voltage.

Fig. 8 shows the simulated waveforms for the practical circuit elements in the case of the $60 \mathrm{~Hz}$ grid frequency, where practical system parameters are used, except for the inductance value. It is assumed that the inductance is changed $20 \%$ from its nominal value, i.e., $\delta=20 \%$, to evaluate the performance of the proposed control scheme under parameter variations. In Fig. 8(a), the estimated voltage error is significantly higher than that of the ideal case shown in Fig. 7 (a). Because the actual value of the inductor is larger than its 


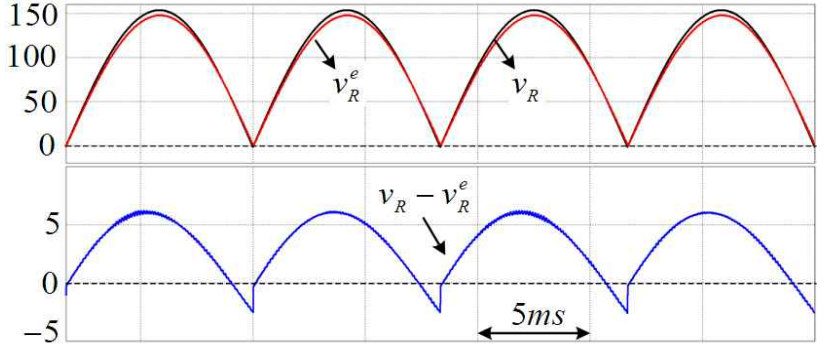

(a)

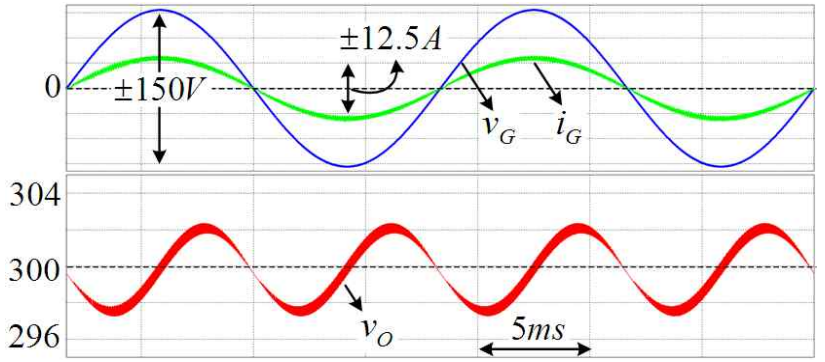

(b)

Fig. 8. Simulated waveforms for practical circuit elements in the case of the $60 \mathrm{~Hz}$ grid. (a) Estimated rectified grid voltage and estimation error. (b) Grid voltage, grid current, and output voltage.
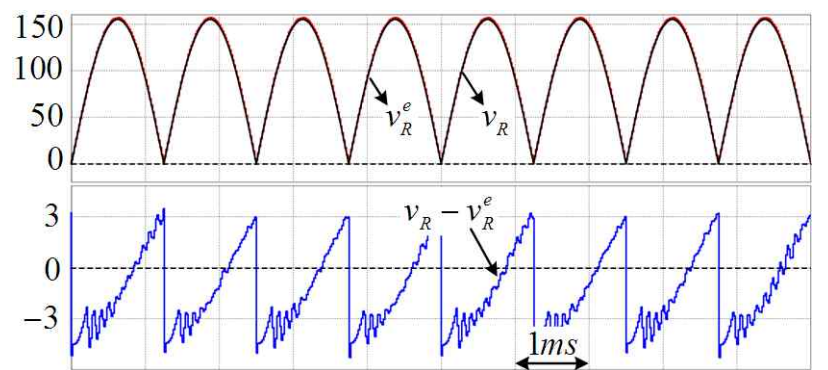

(a)

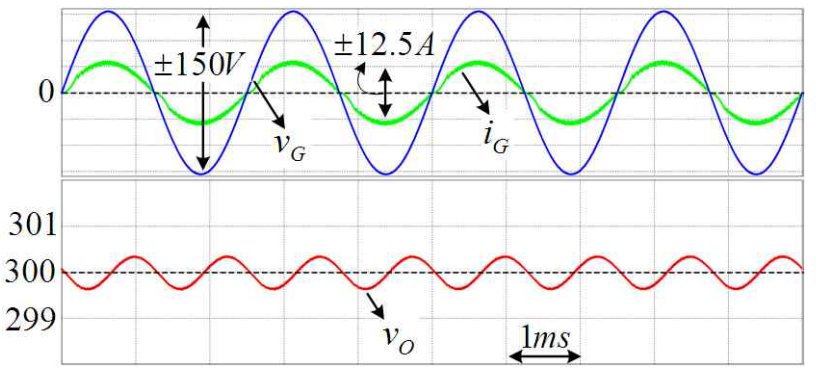

(b)

Fig. 9. Simulated waveforms for ideal circuit elements in the case of the $400 \mathrm{~Hz}$ grid. (a) Estimated rectified grid voltage and error. (b) Grid voltage, grid current, and output voltage.

nominal value, the compensated phase is insufficient to compensate for the phase lag of the switch voltage. However, as shown in Fig. 8(b), the grid current is still adjusted to be a sinusoidal waveform and in phase with the grid voltage. Thus, it can be seen that the proposed control strategy can be applied to practical systems that include inductance variations.

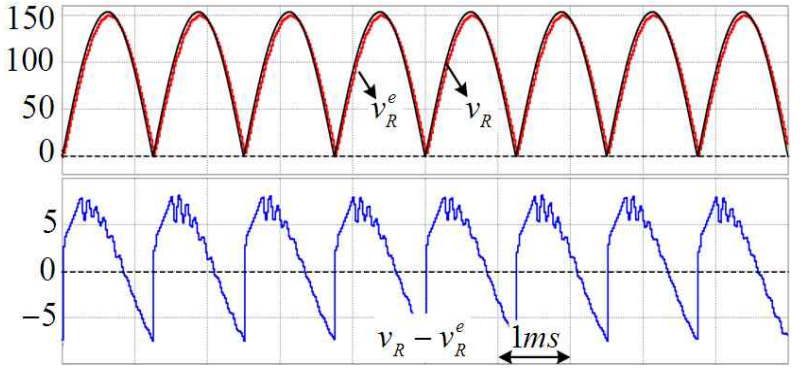

(a)

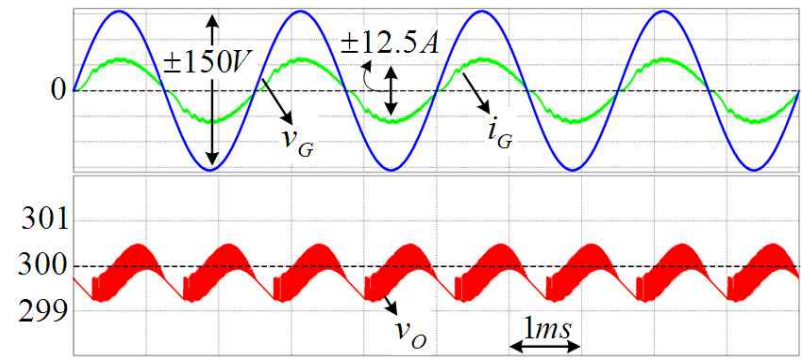

(b)

Fig. 10. Simulated waveforms for practical circuit elements in the case of the $400 \mathrm{~Hz}$ grid. (a) Estimated rectified grid voltage and estimation error. (b) Grid voltage, grid current, and output voltage.

To evaluate the performance of the proposed control scheme for high-grid-frequency applications, an aircraft application was considered, which usually uses a $400-\mathrm{Hz}$ grid power supply. Figs. 9 and 10 show the simulated results, obtained under the same conditions as those used in Figs. 7 and 8 , respectively, except for the grid frequency. Like the $60-\mathrm{Hz}$ case, the rectified grid voltage can be detected successfully by the proposed estimation method. This shows that the proposed control scheme can also be applied to high grid frequencies. However, based on the theoretical analyses, the system performance becomes highly sensitive to variations in the inductance at the high frequency power supply, and the grid current includes small harmonics. However, the system still maintains a near-unity input power factor $(\mathrm{PF}=0.9965)$ despite the degraded performance when compared with the low grid frequency at $60 \mathrm{~Hz}$.

To investigate the effect of inductance variations under practical conditions, the total harmonics distortion (THD) of the grid current is plotted in Fig. 11 according to the inductance variation $\delta(\%)$, for both the $60 \mathrm{~Hz}$ and $400 \mathrm{~Hz}$ grid frequencies. As anticipated, the grid current is distorted more significantly for larger inductance variations. In addition, it can be seen that the $400-\mathrm{Hz}$ grid current is more seriously affected by inductance variations than the $60-\mathrm{Hz}$ grid current. For instance, when $\delta=20 \%$, the THD of the $60-\mathrm{Hz}$ grid current is only $2.16 \%$, whereas the THD in the $400-\mathrm{Hz}$ case is $4 \%$ with the same variation.

\section{EXPERIMENTAL RESULTS}

The proposed control system is implemented by a DSP 


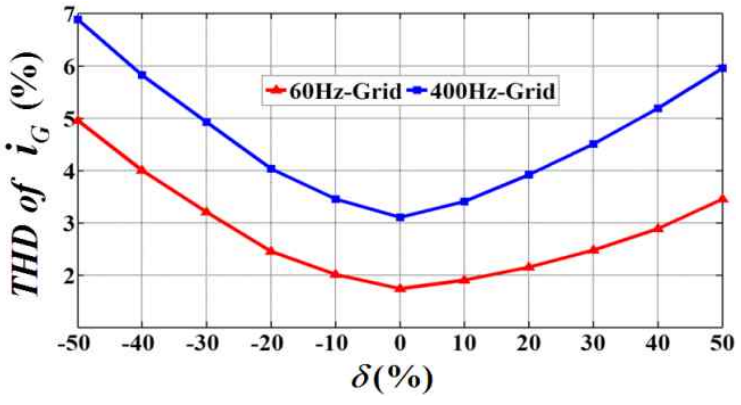

Fig. 11. Simulation results of THD of grid current with respect to the inductance variation levels under practical conditions.

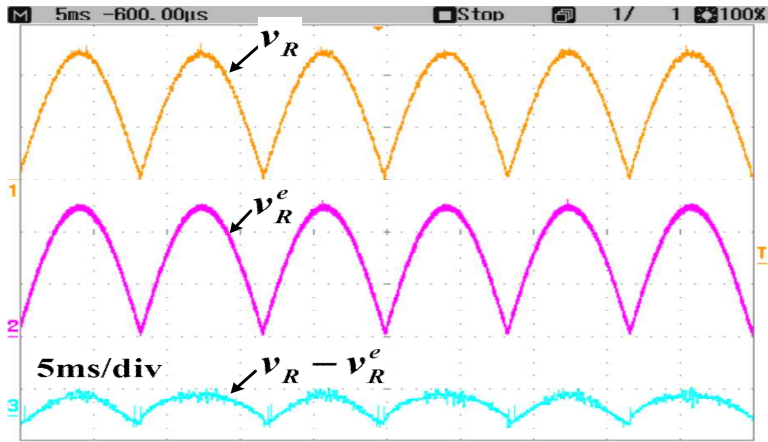

Fig. 12. Experimental result of the rectified grid voltage detection method. Top: rectified grid voltage (70V/div). Middle: detected rectified grid voltage $(70 \mathrm{~V} / \mathrm{div})$. Bottom: The error of the detecting method $(7 \mathrm{~V} / \mathrm{div})$.

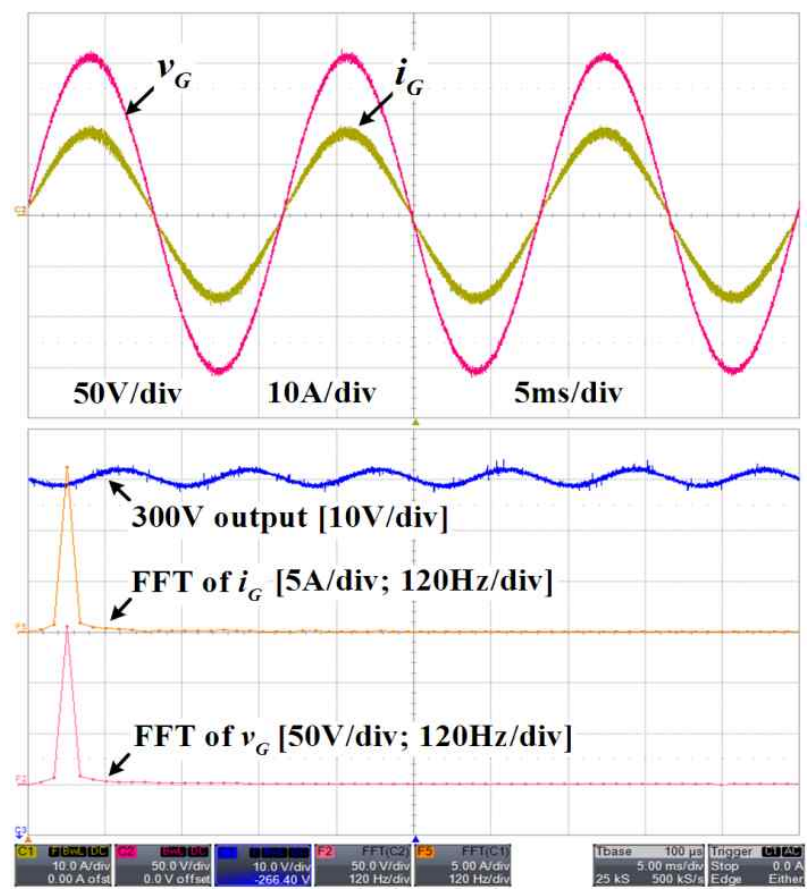

Fig. 13. Experimental results of the system under conditions: 110 $\mathrm{V}_{\mathrm{rms}}-60 \mathrm{~Hz}$ power grid, $300 \mathrm{~V}$ desired output, and $80 \Omega$ load.

(TMS320F28335 from Texas Instruments) to manage a $3 \mathrm{~kW}-\mathrm{PFC}$ boost converter with the same parameters as those used in the simulations. To implement the proposed control scheme, only two sensors are required, an LEM LV25-P for
TABLE II

Measured Data Under $110 \mathrm{~V}_{\text {RMs }}-60 \mathrm{~Hz}$ Power Grid FOR VARIOUS RESISTIVE LOADS

\begin{tabular}{|c|c|c|c|c|}
\hline$R_{\text {Load }}(\Omega)$ & 60 & 80 & 100 & 120 \\
\hline$P_{g}(W)$ & 1600 & 1200 & 950 & 785 \\
\hline$P F$ & 0.9992 & 0.9976 & 0.9959 & 0.9945 \\
\hline$T H D(\%)$ & 1.84 & 2.21 & 2.58 & 2.97 \\
\hline
\end{tabular}

sensing the output voltage and an LEM HX30-P for measuring the grid current. In this experiment, the MOSFET is switched at a $50 \mathrm{kHz}$ frequency and triggered by a HCPL-3120 gate driver. To measure the input power factor and grid current harmonics, a power analyzer HIOKI-3193 is used. In addition, a programmable ac source (Chroma-61704) is utilized to emulate the $60-\mathrm{Hz}$ and $400-\mathrm{Hz}$ power grids.

Fig. 12 shows the experimental results of the rectified grid voltage detection method. The error is near zero when compared with the peak grid voltage. This demonstrates that the proposed detection method is able to identify the grid voltage successfully. The error shown in Fig. 12 is consistent with the simulation results shown in Fig. 7 and 8.

In Fig. 13, the waveforms of the grid voltage and current, the output voltage, and the fast Fourier transforms (FFT) of the grid voltage and current are illustrated. The output voltage successfully tracks the command. However, it inherently contains a double-grid frequency ac ripple component whose magnitude depends on the converter parameters: the smoothing capacitor, grid frequency, and output power [17]. From the grid current waveform and its FFT, it can be seen that the grid current is controlled to be a sinusoidal waveform with a $\mathrm{THD}=2.21 \%$ and a $\mathrm{PF}=0.9976$, which confirms the good performance of the proposed control scheme. To compare the grid current THD with the IEC-61000-3-2 class A and class D standards that regulate the limitation of grid current harmonics, the grid current harmonics are plotted, as shown in Fig. 14. It is clear that the proposed control scheme satisfies the limits required by the IEC-61000-3-2 standard.

In addition, the system is verified under various load conditions: $60 \Omega, 80 \Omega, 100 \Omega$, and $120 \Omega$ with the output voltage regulated at $300 \mathrm{Vdc}$. The measured grid power supply, the input power factor, and the THD of the grid current are listed in Table II. By examining the measured data according to the given loads, it can be concluded that when a load is heavier, the system obtains a higher power factor and a lower grid current harmonic distortion. However, the system still performs a near-unity power factor $(\mathrm{PF}=0.9945)$, and the grid current is a near-pure sinusoidal $(\mathrm{THD}=2.97 \%)$ despite light load operation ( $P_{g} \approx 785 \mathrm{~W}$ ).

The performance of the proposed control scheme for a $400-\mathrm{Hz}$ grid frequency is shown in Fig. 15 under the same 


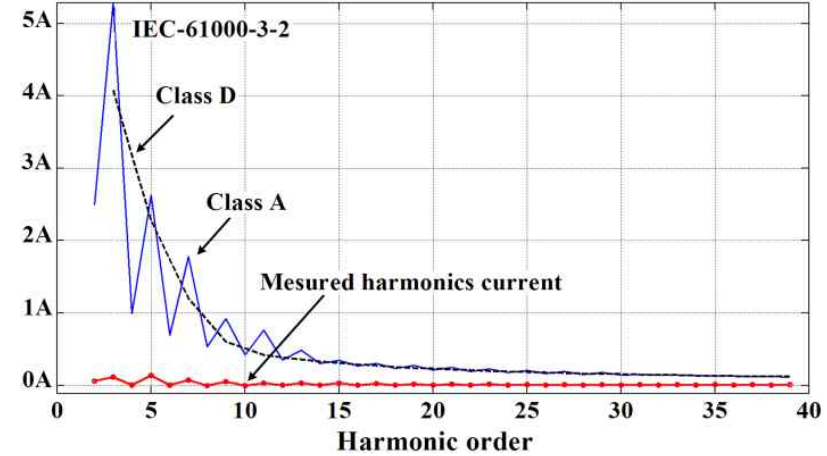

Fig. 14. Measured harmonics current under conditions: 110 $\mathrm{V}_{\mathrm{rms}}-60 \mathrm{~Hz}$ power grid, $300 \mathrm{~V}$ desired output, and $80 \Omega$ load; and within the limits of the IEC-61000-3-2 standard.

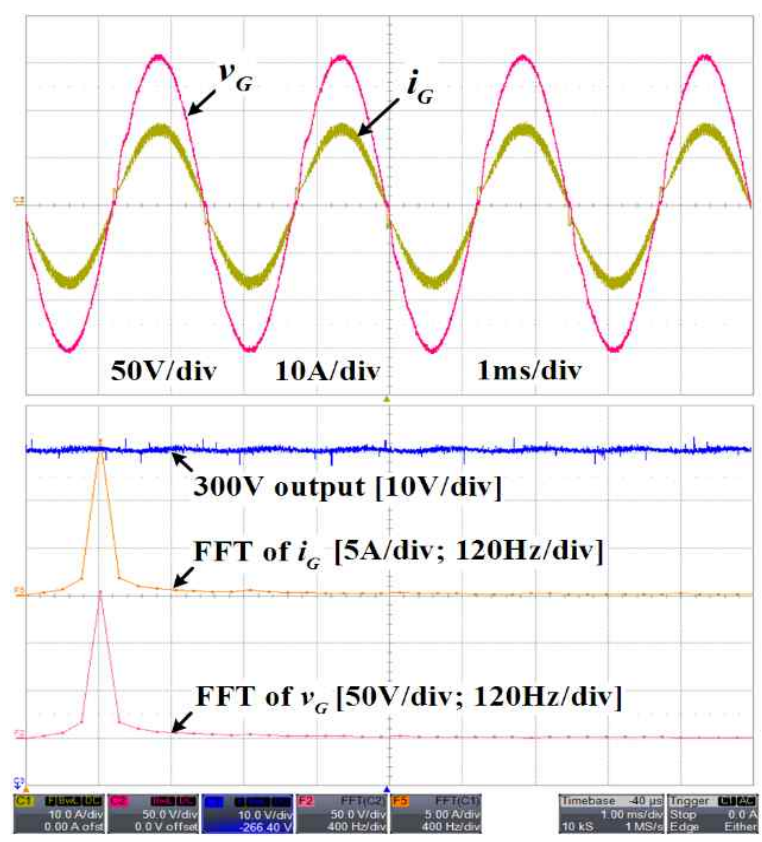

Fig. 15. Experimental results of the system under condition: $110 \mathrm{~V}_{\mathrm{rms}}-400 \mathrm{~Hz}$ power grid, $300 \mathrm{~V}$ desired output, and $80 \Omega$ load.

TABLE III

Measured Data Under 110 VRms-400 Hz Power Grid FOR VARIOUS RESISTIVE LOADS

\begin{tabular}{|c|c|c|c|c|}
\hline$R_{\text {Load }}(\Omega)$ & 60 & 80 & 100 & 120 \\
\hline$P_{g}(W)$ & 1630 & 1210 & 960 & 790 \\
\hline$P F$ & 0.9975 & 0.9949 & 0.9928 & 0.9910 \\
\hline$T H D(\%)$ & 3.52 & 4.05 & 4.53 & 5.01 \\
\hline
\end{tabular}

conditions as those used in the $60-\mathrm{Hz}$ case. It is apparent that the control system injects a sinusoidal grid current with a low harmonic distortion $(\mathrm{THD}=4.05 \%)$ and obtains a high input power factor $(\mathrm{PF}=0.9949)$. In order to confirm the system stability characteristics under various load conditions, we showthe grid power, the input power factor, and the THD of the grid current given in Table III according to several load conditions. As in the case of the $60-\mathrm{Hz}$ grid frequency, a high
PF and low THD performance is obtained over all of the load ranges.

\section{CONCLUSIONS}

A grid-voltage-sensorless control scheme with duty ratio feedback for PFC boost converters has been developed in this paper. It can be easily implemented, and its performance is not seriously affected by variations of the converter components. In particular, duty-ratio feedback is applied to the current controller to improve the control performance. This makes the grid current to be a sinusoidal waveform in phase with the grid voltage. The proposed control scheme is designed and analyzed by using a small-signal model of the converters for typical commercial grid frequencies, $60 \mathrm{~Hz}$ and $400 \mathrm{~Hz}$. Its feasibility is evaluated by simulation and experiments together with a mathematical analysis. Furthermore, it is simple to implement the proposed control scheme. Finally, the proposed control scheme has a strong chance in the commercial applications.

\section{ACKNOWLEDGMENT}

This work was supported by the National Research Foundation of Korea Grant funded by the Korean Government (No. 2010-0025483).

\section{REFERENCES}

[1] J. C. Crebier, B. Revol, and J. P. Ferrieux, "Boost-chopper-derived PFC rectifiers: Interest and reality," IEEE Trans. Ind. Electron., Vol. 52, No. 1, pp. 36-45, Feb. 2005.

[2] O. Garcia, J. A. Cobos, R. Prieto, P. Alou, and J. Uceda, "Single phase power factor correction: A survey," IEEE Trans. Power Electron., Vol. 18, No. 3, pp. 749-754, May 2003.

[3] J. C. Gomez and M. M. Morcos, "Impact of EV battery chargers on the power quality of distribution systems," IEEE Trans. Power Del., Vol. 18, No. 3, pp. 975-981, Jul. 2003.

[4] "Limits for harmonic current emissions," International Electro-technical Commission Standard 61000-3-2, 2004.

[5] "Environmental conditions and test procedures for airborne equipment," Tech. Rep., RTCA DO-160D, 2001.

[6] X. Xiaogao, C. Zhao, L. Qiang, and L. Shirong, "A novel integrated buck-flyback nonisolated PFC converter with high power factor," IEEE Trans. Ind. Electron., Vol. 60, No. 12, pp. 5603-5612, Dec. 2013.

[7] A. A. Fardoun, E. H. Ismail, A. J. Sabzali, and M. A. Al-Saffar, "New efficient bridgeless Cuk rectifiers for PFC applications," IEEE Trans. Power Electron., Vol. 27, No. 7, pp. 3292-3301, Jul. 2012.

[8] Y. S. Kim, W. Y. Sung, and B. K. Lee, "Comparative performance analysis of high density and efficiency PFC topologies," IEEE Trans. Power Electron., Vol. 29, No. 6, pp. 2666-2679, Jun. 2014.

[9] F. Musavi, M. Edington, W. Eberle, and W. G. Dunford, "Evaluation and efficiency comparison of front end AC-DC plug-in hybrid charger topologies," IEEE Trans. Smart Grid, 
Vol. 3, No. 1, pp. 413-421, Mar. 2012.

[10] M. H. Chan, Y. T. Lin, and Y. Y. Tzou, "Development of a mixed-signal PFC control IC with fast dynamic response," in Proc. Power Electronics and Motion Control Conference, 2009, pp. 1708-1712, May 2009.

[11] A. E. Aroudi, R. Haroun, A. C. Pastor, and L. M. Salamero, "Suppression of line frequency instabilities in PFC ac-dc power supplies by feedback notch filtering the pre-regulator output voltage," IEEE Trans. Circuits Syst. I, Reg. Papers, Vol. 60, No. 3, pp. 796-809, Mar. 2013.

[12] W. Ma, M. Wang, S. Liu, S. Li, and P. Yu, "Stabilizing the average current-mode-controlled boost PFC converter via washout-filter-aided method," IEEE Trans. Circuits Syst. II, Exp. Briefs, Vol. 58, No. 9, pp. 595-599, Sep. 2011.

[13] J. Sun, "On the zero-crossing distortion in single-phase PFC converters," IEEE Trans. Power Electron., Vol. 19, No. 3, pp. 685-692, May 2004.

[14] D. M. Van, K. D. Gusseme, A. P. M. V. denBossche, and J. A. Melkebeek, "Duty-ratio feedforward for digitally controlled boost PFC converters," IEEE Trans. Ind. Electron., Vol. 52, No. 1, pp. 108-115, Jan. 2005.

[15] M. Chen and J. Sun, "Feedforward current control of boost single-phase PFC converters," IEEE Trans. Power Electron., Vol. 21, No. 2, pp. 338-345, Mar. 2006.

[16] T. Ohnishi and M. Hojo, "DC voltage sensorless single-phase PFC converter," IEEE Trans. Power Electron., Vol. 19, No. 2, pp. 404-410, Mar. 2004.

[17] V. M. Lopez, F. J. Azcondo, A. Castro, and R. Zane, "Universal digital controller for boost CCM power factor correction stages based on current rebuilding concept," IEEE Trans. Power Electron., Vol. 29, No. 7, pp. 3818-3829, Jul. 2014.

[18] M. Pahlevaninezhad, S. Pan, S. Eren, A. Bakhshai, and P. Jain, "An adaptive nonlinear current observer for boost PFC AC/DC converters," IEEE Trans. Ind. Electron., to be published.

[19] F. J. Azcondo, A. D. Castro, V. M. Lopez, and O. Garcia, "Power factor correction without current sensor based on digital current rebuilding," IEEE Trans. Power Electron., Vol. 25, No. 6, pp. 1527-1536, Jun. 2010.

[20] H. C. Chen, C. C. Lin, and J. Y Liao, "Modified single-loop current sensorless control for single-phase boost-type SMR with distorted input voltage," IEEE Trans. Power Electron., Vol. 26, No. 5, pp. 1322-1328, May 2011.

[21] B. A. Mather and D. Maksimović, "A simple digital power-factor correction rectifier controller," IEEE Trans. Power Electron., Vol. 26, No. 1, pp. 9-19, Jan. 2011.

[22] J. H. Chiang, Bin-Da Liu, and S. M. Chen, "A simple implementation of nonlinear-carrier control for power factor correction rectifier with variable slope ramp on field-programmable gate array," IEEE Trans. Ind. Informatics, Vol. 9, No. 3, pp. 1322-1329, Aug. 2013.

[23] M. K. Kazimierczuk, "Pulse-width modulated DC-DC power converters," Jonh Wiley \& Sons Publication, 1st Edition, Chap. 10, 2008.

[24] D. M. Van de Sype, K. De Gussemé, and J. A. Melkebeek, "Frequency domain analysis of digital pulse-width modulators," in Proc. Electrimacs'02, 2002.

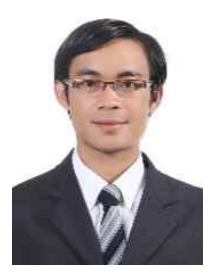

Cong-Long Nguyen was born in Nghe-An Province, Vietnam. He received his B.S. degree in Electrical Engineering from the University of Technology, Ho Chi Minh City, Vietnam, in 2010. He worked for Intel Corporation as a Production Engineer in Vietnam site. He is presently a Research Assistant at the University of Ulsan, Ulsan, Korea, where he is presently working toward his Ph.D. degree in Electrical Engineering. His current research interests include power electronics applied to electric vehicles, power quality control, and the optimization of electric energy storage in renewable energy conversion systems.

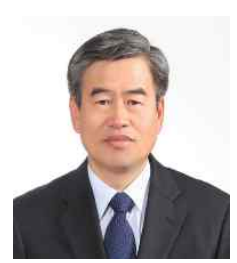

Hong-Hee Lee received his B.S., M.S., and $\mathrm{Ph} . \mathrm{D}$. degrees in Electrical Engineering from Seoul National University, Seoul, Korea, in 1980, 1982, and 1990, respectively. From 1994 to 1995 , he was a Visiting Professor at Texas A\&M University, College Station, TX, USA. Since 1985, he has been with the Department of Electrical Engineering, University of Ulsan, Ulsan, Korea, where he is presently a Professor in the School of Electrical Engineering. $\mathrm{He}$ is also the Director of the Network-based Automation Research Center (NARC), which is sponsored by the Ministry of Knowledge Economy (MKE). His current research interests include power electronics, network-based motor control, and control networks. He is a Member of the Institute of Electrical and Electronics Engineers (IEEE), the Korean Institute of Power Electronics (KIPE), the Korean Institute of Electrical Engineers (KIEE), and the Institute of Control, Robotics and Systems (ICROS).

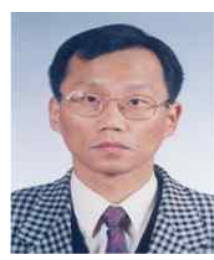

Tae-Won Chun was born in Korea, in 1959. He received his B.S. degree in Electrical Engineering from Pusan National University, Pusan, Korea, in 1981, and his M.S. and Ph.D degrees in Electrical Engineering from Seoul National University, Seoul, Korea, in 1983 and 1987, respectively. Since 1986, he has been a faculty member in the Department of Electrical Engineering, Ulsan University, Ulsan, Korea, where he is presently a Full Professor. He was a Visiting Scholar with the Department of Electrical and Computer Engineering, University of Tennessee, Knoxville, TN, USA. From 2005 to 2006, he served as a Visiting Scholar with the Department of Electrical and Computer Engineering, Virginia Polytechnic Institute and State University, Blacksburg, VA, USA. His current research interests include grid-connected inverter systems and ac motor control. 